http://dx.doi.org/10.1590/1678-4162-6885

Arq. Bras. Med. Vet. Zootec., v.67, n.3, p.899-908, 2015

\title{
Modelagem das trajetórias médias do peso vivo e do rendimento de carcaça de tilápias do Nilo (Oreochromis niloticus) por meio de funções B-spline
}

\author{
[Modeling the mean trajectories of live body weight and carcass yield of Nile tilapia \\ (Oreochromis niloticus) by B-spline functions] \\ D.C.C. Santos ${ }^{1}$, D.C.B. Scalez ${ }^{2}$, T.L. Passafaro ${ }^{1}$, E.M. Turra ${ }^{1}$, A.F.A. Fernandes ${ }^{1}$, \\ E.R. Alvarenga ${ }^{1}$, G.F.O. Alves ${ }^{1}$, F.L.B. Toral ${ }^{1}$ \\ ${ }^{1}$ Universidade Federal de Minas Gerais - Belo Horizonte, MG \\ ${ }^{2}$ Universidade Estadual Paulista - Jaboticabal, SP
}

\begin{abstract}
RESUMO
Empregando o método dos quadrados mínimos e polinômios B-spline quadráticos, diferentes modelos estatísticos foram testados para identificar o mais apropriado para modelar as trajetórias médias do peso vivo e do rendimento de carcaça de tilápias do Nilo (Oreochromis niloticus). Dados de peso vivo (8.758) e de rendimento de carcaça (2.042) de tilápias com idades entre 106 e 245 dias foram obtidos de 72 famílias provenientes de 36 machos e 72 fêmeas. As variáveis sexo e tanque de criação foram consideradas como classificatórias, e os coeficientes dos polinômios B-spline quadráticos com dois a cinco intervalos de mesmo tamanho foram utilizados como covariáveis. Segundo a maioria dos critérios de ajuste utilizados, os modelos com polinômio B-spline quadrático com cinco intervalos de mesmo tamanho apresentaram os melhores ajustes. $\mathrm{O}$ aumento do número de intervalos do polinômio B-spline melhorou o ajuste dos polinômios aos dados. A inclusão dos efeitos classificatórios de sexo, tanque de criação, interação entre esses efeitos e polinômio B-spline quadrático aninhado a essa interação indicou que, com o decorrer do tempo, cada sexo, cultivado em diferente tanque, apresentou trajetória média diferente, sendo necessária a inclusão do aninhamento do tempo na interação sexo x tanque de criação para que, em programas de melhoramento genético da espécie, os valores genéticos dos candidatos à seleção não sejam sub ou superestimados.
\end{abstract}

Palavras-chave: ajuste de modelos, aninhamento, melhoramento genético, quadrados mínimos, polinômios

\begin{abstract}
Employing the method of least squares and quadratic B-spline polynomials, different statistical models were tested to identify the most appropriate to model the mean trajectories of live weight and carcass yield of Nile tilapia (Oreochromis niloticus). Data of live weight $(8,758)$ and carcass yield $(2,042)$ of tilapias with ages between 106 and 245 days were obtained from 72 families derived from 36 males and 72 females. The sex and tank variables were considered as classificatory and the coefficients of quadratic polynomials B-spline of two to five intervals of the same size were used as covariables. According to most fit criteria used, the models with quadratic B-spline polynomial with five intervals of the same size presented the best adjustments. The increase in number of intervals of B-spline polynomial improved the fit of the polynomials to the data. The inclusion of classificatory effects from sex, tank, the interaction of these effects and the quadratic polynomial B-spline nested in this interaction indicated that, over time, each sex, grown in different tank, presented different mean trajectory, necessitating the inclusion of nesting time in the interaction sex $x$ tank in order to avoid the under or overestimation of breeding values of the selection candidates in breeding programs of this species.
\end{abstract}

Keywords: fit of models, genetic breeding, least squares, nesting, polynomials

Recebido em 23 de maio de 2013

Aceito em 12 de março de 2015

E-mail: dalinne_chrystian@hotmail.com 


\section{INTRODUÇÃO}

A maior comercialização da tilápia (Oreochromis niloticus) resultou na procura por animais de melhor desempenho produtivo (Santos et al., 2007b) e no interesse pelo melhoramento genético da espécie (Rutten et al., 2005).

O peso vivo ao abate e o rendimento de carcaça, características diretamente ligadas ao retorno econômico, são importantes para os produtores e programas de melhoramento genético da espécie (Santos et al., 2012). Essas características podem ser estudadas por meio dos modelos de regressão aleatória. Tais modelos admitem a utilização de polinômios B-spline, que têm sido propostos como alternativa para ajustar as funções de covariância e modelar as trajetórias médias de crescimento (Menezes et al., 2011).

A utilização de modelos estatísticos e metodologias de avaliação inadequados levam à queda da acurácia e podem reduzir o progresso genético (Valente et al., 2008). Além disso, o tipo e a ordem de ajuste dos polinômios utilizados para modelar as trajetórias médias de crescimento podem influenciar o ajuste das funções de covariâncias e os resultados das avaliações genéticas (Toral et al., 2014). Para a obtenção de valores genéticos, existem diferentes modelos de regressão aleatória utilizando polinômios B-spline que diferem quanto ao grau, posição dos nós, número de intervalos e classes de variância residual. A escolha do modelo com melhor ajuste acarretará na predição de valores genéticos e resposta à seleção adequadas.

Sendo assim, este trabalho foi realizado com o objetivo de identificar o modelo mais apropriado para modelar as trajetórias médias do peso vivo e do rendimento de carcaça de tilápias do Nilo, utilizando-se polinômios do tipo B-spline quadráticos.

\section{MATERIAL E MÉTODOS}

Foi utilizada base de dados constituída de 72 famílias de irmãos completos e 36 famílias de meio-irmãos de tilápias do Nilo (Oreochromis niloticus) provenientes do Laboratório de Aquacultura da Escola de Veterinária da Universidade Federal de Minas Gerais. As 72 famílias de irmãos completos e as 36 famílias de meio-irmãos foram formadas utilizando 72 fêmeas e 36 machos, sendo acasalado um macho com duas fêmeas. Por falta de espaço físico suficiente e devido às desovas assíncronas das fêmeas, todas as famílias foram formadas em período de 17 semanas (julho a novembro de 2009). Depois de realizada marcação individual com microchips (Microchip, Partners \& Quality Technology, Brasil) e sexagem dos indivíduos, entre a $13^{\mathrm{a}}$ e a $20^{\mathrm{a}}$ semana de vida pós-eclosão das larvas, grupos de seis famílias, mais próximas em idade, foram alojadas comunalmente em tanques circulares de $7 \mathrm{~m}^{3}$. Devido às sobrevivências desiguais, o número de animais marcados por família foi diferente. Para garantir a mesma densidade de estocagem (aproximadamente 29 indivíduos $/ \mathrm{m}^{3}$ ), peixes não identificados, excedentes de famílias, foram alojados em tanques com menor número de tilápias. As 72 famílias ocuparam 12 tanques de mesma dimensão e, para reduzir diferenças entre tanques, as mesmas condições de cultivo foram impostas. Informações sobre manejo e condições de cultivo foram apresentadas em Turra et al. (2012).

Indivíduos de cada família foram pesados em uma até seis oportunidades, sendo a primeira pesagem realizada no momento da identificação. As idades de pesagens foram diferentes para cada família e, no total, foram obtidas 62 idades de avaliação. A cada mensuração, depois de submetidos à crionarcose, alguns animais por família foram aleatoriamente escolhidos para realização do abate, pesagem da carcaça e confirmação do sexo. No sexto momento de mensuração, todos os animais remanescentes de cada família foram abatidos. A carcaça, obtida manualmente por única pessoa, foi considerada o animal sacrificado, sem cabeça, cintura pélvica, cintura torácica (corte oblíquo da cabeça) e vísceras, mas mantidas as escamas. O rendimento de carcaça foi obtido pela divisão do peso da carcaça pelo peso vivo do indivíduo. $\mathrm{O}$ intervalo de dias entre cada mensuração variou de 14 a 28 dias e, dessa forma, os dados das pesagens foram distribuídos ao longo do período de 106 a 245 dias de idade.

O banco de dados foi constituído de 8.758 medidas de peso vivo e 2.042 observações para rendimento de carcaça. Dos 2.042 indivíduos avaliados para peso vivo, 230 (11,26\%), 242 $(11,85 \%), 228(11,17 \%), 228(11,17 \%), 236$ 
$(11,56 \%)$ e $878 \quad(43,00 \%)$ obtiveram, respectivamente, uma, duas, três, quatro, cinco e seis observações.

As trajetórias médias do peso vivo e do rendimento de carcaça foram modeladas por meio de polinômios do tipo B-splines quadráticos com número total de intervalos do mesmo tamanho variando entre 2 e 5 . Além disso, foram consideradas alternativas para modelagem dos efeitos classificatórios de sexo e tanque de criação dos animais. De forma geral, os modelos considerados podem ser representados pelas equações:

$$
\begin{aligned}
& y_{h i j l}=\mu+s_{h}+t_{i}+\sum_{k=2}^{K} \phi_{k, 2}\left(d_{j}\right) \beta_{k}+e_{h i j l}(\text { modelo S+T }+\mathrm{B}), \\
& y_{h i j l}=\mu+s t_{(h i)}+\sum_{k=2}^{K} \phi_{k, 2}\left(d_{j}\right) \beta_{k}+e_{h i j l}(\operatorname{modelo} \mathrm{ST}+\mathrm{B}), \\
& y_{h i j l}=\mu+s_{h}+t_{i}+\sum_{k=2}^{K} \phi_{k, 2}\left(d_{j}\right)_{(h)} \beta_{k_{(h)}}+e_{h i j l}(\operatorname{modelo} \mathrm{S}+\mathrm{T}+\mathrm{B}(\mathrm{S})), \\
& y_{h i j l}=\mu+s_{h}+t_{i}+\sum_{k=2}^{K} \phi_{k, 2}\left(d_{j}\right)_{(i)} \beta_{k_{(i)}}+e_{h i j l}(\operatorname{modelo~S}+\mathrm{T}+\mathrm{B}(\mathrm{T})) \mathrm{e}, \\
& y_{h i j l}=\mu+s t_{(h i)}+\sum_{k=2}^{K} \phi_{k, 2}\left(d_{j}\right)_{(h i)} \beta_{k_{(h i)}}+e_{h i j l}(\operatorname{modelo~ST}+\mathrm{B}(\mathrm{ST})),
\end{aligned}
$$

em que: $y_{\text {hijl }}=$ valor observado da variável dependente $y$ da tilápia $l$, do sexo $h$, no tanque $i$, mensurada na $j$-ésima idade; $\mu=$ constante geral; $S_{h}=$ efeito classificatório do sexo $h(h=1,2) ; t_{i}=$ efeito classificatório do tanque $i(i=1, \ldots, 12) ; \phi_{k, 2}\left(d_{j}\right), \phi_{k, 2}\left(d_{j}\right)_{(h)}$, $\phi_{k, 2}\left(d_{j}\right)_{(i)}, \phi_{k, 2}\left(d_{j}\right)_{(h i)}=k$-ésimas covariáveis para as $j$-ésimas idades, sem aninhamento, com aninhamento em sexo, com aninhamento em tanque de criação e com aninhamento na interação sexo $\mathrm{x}$ tanque, obtidas por meio de polinômios B-spline quadráticos, respectivamente; $\beta_{k}, \beta_{k_{(h)}}, \beta_{k_{(i)}}, \beta_{k_{(h i)}}=k$ ésimos coeficientes de regressão gerados para os polinômios B-spline quadráticos apresentando até cinco intervalos $(K=2, \ldots, 5) ; e_{h i l}=$ erro aleatório associado a cada observação. Nas representações simbólicas dos modelos, S é o efeito classificatório de sexo; T é o efeito classificatório de tanque de criação; ST são os efeitos classificatórios de sexo, tanque e interação sexo $\mathrm{x}$ tanque; $\mathrm{B}$ é a covariável obtida com polinômio B-spline sem aninhamento; B(S), $\mathrm{B}(\mathrm{T})$ e $\mathrm{B}(\mathrm{ST})$ são as covariáveis obtidas com polinômio B-spline com aninhamento em sexo, em tanque $\mathrm{e}$ na interação sexo $\mathrm{x}$ tanque, respectivamente.

Os coeficientes das funções B-spline foram definidos para o intervalo $k$, delimitado pelos pontos definidos pelas idades $\mathrm{D}_{k}$ e $\mathrm{D}_{k+1}$, em que $\mathrm{D}_{k}<\mathrm{D}_{k+1}$. Para funções B-spline de grau $p$ igual a zero $(p=0)$, todos os coeficientes para as idades $d_{j}$ presentes dentro do intervalo $k$ especificado foram iguais à unidade $\left(\phi_{k, p=0}\left(d_{j}\right)=1\right.$, se $\left.D_{k} \leq d_{j}<D_{k+1}\right)$. Os coeficientes para as idades $d_{j}$ que se apresentaram fora do intervalo $k$ receberam valores iguais a zero $\left(\phi_{k, p=0}\left(d_{j}\right)=0\right.$, de outra maneira). Para funções B-spline de grau $p$ maior que zero $(p>0)$, os coeficientes $\phi_{k, p}\left(d_{j}\right)$ foram gerados de maneira recursiva pela seguinte função:

$$
\phi_{k, p}\left(d_{j}\right)=\frac{d_{j}-D_{k}}{D_{k+p}-D_{k}} \phi_{k, p-1}\left(d_{j}\right)+\frac{D_{k+p+1}-d_{j}}{D_{k+p+1}-D_{k+1}} \phi_{k+1, p-1}\left(d_{j}\right) \text {. }
$$


No presente estudo, os coeficientes das funções B-spline foram gerados por meio de funções quadráticas $(p=2)$ que incluíram até cinco intervalos do mesmo tamanho. Polinômios Bspline de grau 2 foram escolhidos para modelagem das trajetórias médias do peso vivo e rendimento de carcaça, pois têm se mostrado adequados para descrever a estrutura de covariâncias de vários arquivos de dados com resultados plausíveis (Meyer, 2005; Baldi et al., 2010; Boligon et al., 2012). A divisão em $m$ intervalos exigiu a especificação de $m-1$ nós internos e dois nós externos $\left(\mathrm{D}_{0}\right.$ e $\left.\mathrm{D}_{m}\right)$, gerando um total de $m+1$ nós e $m+p$ funções $\phi_{k, p}$ não nulas. Os polinômios B-spline para os modelos estatísticos estudados foram gerados utilizando a função $b s$ do pacote splines do programa estatístico R ( $R$ Development Core Team, versão 2.15.0).

As soluções para os efeitos classificatórios e os coeficientes de regressão para os modelos estatísticos foram obtidos pelo Método dos Quadrados Mínimos por meio da função $l m$ do pacote stats do programa estatístico $\mathrm{R}(R$ Development Core Team, versão 2.15.0). Após obtenção dos coeficientes de regressão, os pesos vivos e rendimentos de carcaça estimados foram obtidos, bem como seus respectivos resíduos. Para a comparação dos modelos, os seguintes critérios de ajuste foram utilizados: Coeficiente de determinação $\left(R^{2}\right)$ : obtido por meio da correlação, elevada ao quadrado, entre valores observados e estimados, para as características peso vivo e rendimento de carcaça. Coeficiente de determinação ajustado $\left(R_{a}^{2}\right)$ : obtido pela fórmula $R_{a}^{2}=1-\frac{n-1}{n-(c+1)}\left(1-R^{2}\right)$, em que: $n$ representa o número de observações no conjunto de dados, $c$ representa o número de coeficientes de regressão utilizados para o ajuste do modelo e $R^{2}$, o coeficiente de determinação do modelo. Desvio médio absoluto (DMA): somatório do módulo da diferença entre valores observados $\left(y_{h i j l}\right)$ e estimados $\left(\hat{y}_{h i j l}\right)$ para peso vivo e rendimento de carcaça, divididos pelos respectivos números de observações $(n)$ dessas características, DMA $=\frac{\sum_{i=1}^{n}\left|y_{h i j l}-\hat{y}_{h i j l}\right|}{n}$.

Soma de Quadrados dos Resíduos (SQR): somatório do quadrado dos erros estimados

( $\left.\boldsymbol{e}_{h i j l}\right)$ para cada observação de peso vivo e rendimento de carcaça, $\mathrm{SQR}=\sum_{i=1}^{n}\left(\hat{e}_{h i j l}\right)^{2}$.

Porcentagem de estimativas de parâmetros significativos do modelo (PPS): divisão entre o número de estimativas de parâmetros significativos do modelo (NPS) e o número de parâmetros do modelo (NP). Também foi realizada a análise gráfica das trajetórias médias do peso vivo e rendimento de carcaça. Modelos com melhores ajustes apresentaram maiores valores para $R^{2}, R_{a}^{2}$ e PPS, e menores valores para DMA e SQR.

\section{RESULTADOS E DISCUSSÃO}

As médias de peso vivo e de rendimento de carcaça variaram dentro do intervalo de idades de avaliação de acordo com o sexo e o tanque de criação dos animais (Fig. 1). Pode-se notar que os tanques de criação não foram avaliados em todas as idades de mensuração dos animais, o que ocorreu pelo fato de as famílias terem sido avaliadas em diferentes oportunidades.

As médias de peso vivo, para fêmeas e machos, no decorrer das idades, variaram entre os tanques, com alguns apresentando valores médios sempre superiores àqueles obtidos em outros tanques (Fig. 1). Em função de o laboratório não apresentar quantidade suficiente de tanques, não foi possível a formação de todas as famílias ao mesmo tempo, sendo as mesmas formadas em período de 17 semanas. Sendo assim, mesmo com condições de cultivo controladas, os efeitos de tanque podem refletir efeitos de alterações ambientais ocorridas ao longo do período de formação das famílias.

As médias do rendimento de carcaça das fêmeas variaram entre $46,54 \%$ e $63,28 \%$ e, para machos, variaram de $52,20 \%$ a $63,15 \%$ (Fig. 1). O processo de obtenção do rendimento de carcaça pode sofrer variações de origem não genética que podem sub ou superestimar o valor fenotípico 
dos candidatos à seleção. Entretanto, tal processo foi realizado manualmente por apenas um técnico, o que pode explicar valores próximos de média de rendimento de carcaça obtidos em diferentes tanques e diferentes idades. Silva et al. (2009), trabalhando com tilápias do Nilo em quatro faixas de peso diferentes $(250$ a $300 \mathrm{~g}, 350$ a $400 \mathrm{~g}, 450$ a $500 \mathrm{~g}$ e 550 a $600 \mathrm{~g}$ ), não obtiveram diferença entre os valores de rendimento de carcaça para essas categorias de peso, cuja média geral foi de $59,10 \%$ e coeficiente de variação de $3,56 \%$. No presente estudo, os valores de média de rendimento de carcaça nessas mesmas faixas de peso foram, respectivamente, de 58,69\%, $59,39 \%, 60,28 \%$ e $61,68 \%$, com média geral de $60,01 \%$ e coeficiente de variação de 4,69\%; valores próximos aos encontrados por Silva et al. (2009).

\section{Fêmeas}

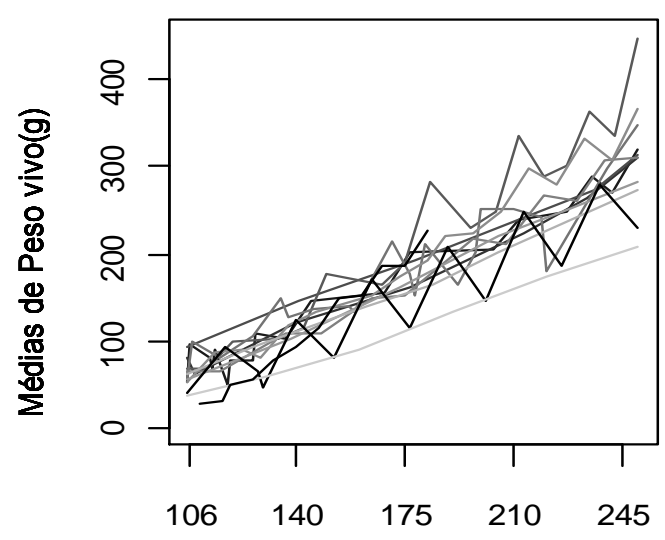

Idade (dias)

\section{Fêmeas}

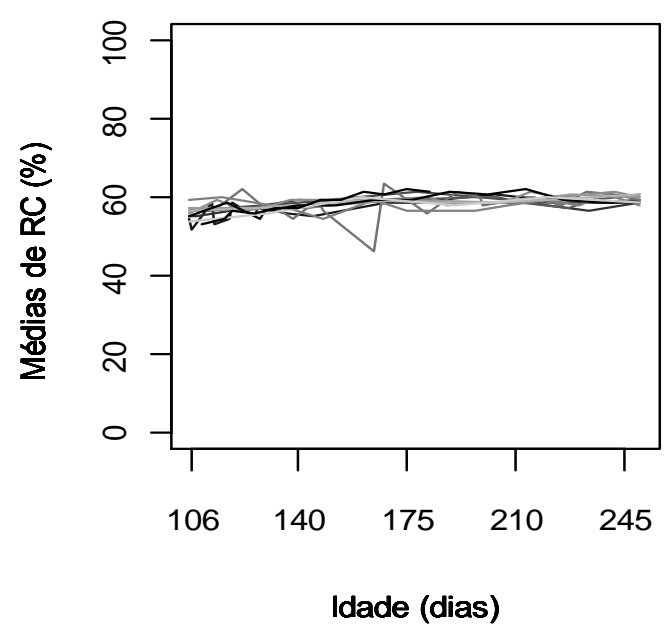

\section{Machos}

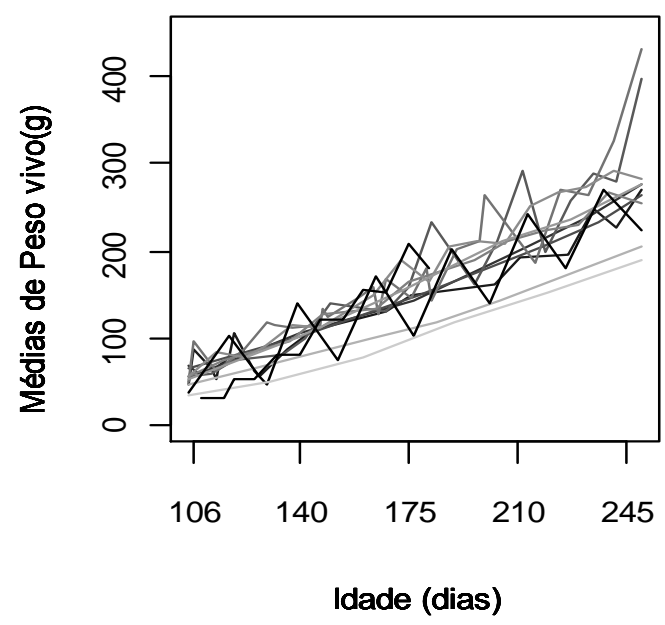

Machos

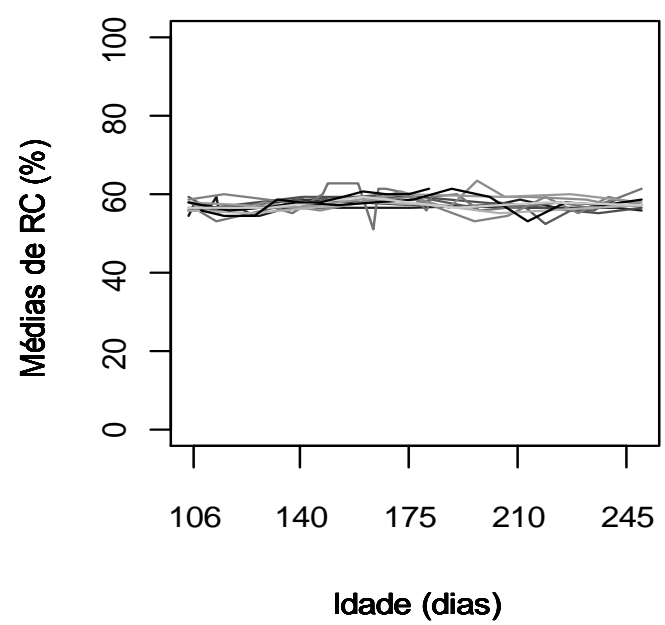

Figura 1. Médias de peso vivo e rendimento de carcaça (RC) de fêmeas e machos de tilápias do Nilo (Oreochromis niloticus) no intervalo de 106 a 245 dias de idade para cada tanque de criação (cada linha de cor diferente representa um tanque de criação). 
De maneira geral, houve pequena variação para os critérios SQR, DMA, $R^{2}$ e $R_{a}^{2}$ de acordo com os diferentes modelos estatísticos testados para a característica peso vivo (Tab. 1). As diferenças entre os maiores e menores valores para esses critérios foram, respectivamente, de $0,188 \mathrm{~g}^{2}, 1,83 \mathrm{~g}, \quad 0,0237$ e 0,0188. Maiores diferenças foram observadas para número de parâmetros (NP, de 16 a 150), e de parâmetros significativos (NPS, de 13 a 30), em que modelos sem aninhamento do polinômio B-spline (modelos $\mathrm{S}+\mathrm{T}+\mathrm{B}$ e $\mathrm{ST}+\mathrm{B})$ obtiveram maior porcentagem de parâmetros significativos (PPS).

Tabela 1. Estatísticas de ajuste ${ }^{1}$ de polinômios B-splines quadráticos para a trajetória média do peso vivo de tilápias do Nilo (Oreochromis niloticus)

\begin{tabular}{|c|c|c|c|c|c|c|c|c|}
\hline Modelo $^{2}$ & $\mathrm{NI}$ & NP & NPS & PPS & SQR & DMA & $R^{2}$ & $R_{a}^{2}$ \\
\hline \multirow{4}{*}{$\mathrm{S}+\mathrm{T}+\mathrm{B}$} & 2 & 16 & 13 & 81 & 2,703 & 41,26 & 0,6574 & 0,6568 \\
\hline & 3 & 17 & 14 & 82 & 2,699 & 41,24 & 0,6579 & 0,6572 \\
\hline & 4 & 18 & 15 & 83 & 2,691 & 41,18 & 0,6589 & 0,6582 \\
\hline & 5 & 19 & 16 & 84 & 2,690 & 41,17 & 0,6590 & 0,6583 \\
\hline \multirow{4}{*}{$\mathrm{ST}+\mathrm{B}$} & 2 & 27 & 25 & 93 & 2,673 & 40,99 & 0,6611 & 0,6601 \\
\hline & 3 & 28 & 26 & 93 & 2,670 & 40,97 & 0,6616 & 0,6606 \\
\hline & 4 & 29 & 27 & 93 & 2,662 & 40,91 & 0,6626 & 0,6615 \\
\hline & 5 & 30 & 28 & 93 & 2,661 & 40,90 & 0,6627 & 0,6616 \\
\hline \multirow{4}{*}{$\mathrm{S}+\mathrm{T}+\mathrm{B}(\mathrm{S})$} & 2 & 19 & 15 & 79 & 2,671 & 40,99 & 0,6614 & 0,6607 \\
\hline & 3 & 21 & 16 & 76 & 2,667 & 40,96 & 0,6619 & 0,6611 \\
\hline & 4 & 23 & 15 & 65 & 2,660 & 40,90 & 0,6628 & 0,6620 \\
\hline & 5 & 25 & 16 & 64 & 2,659 & 40,89 & 0,6629 & 0,6620 \\
\hline \multirow{4}{*}{$\mathrm{S}+\mathrm{T}+\mathrm{B}(\mathrm{T})$} & 2 & 49 & 17 & 35 & 2,601 & 40,29 & 0,6704 & 0,6685 \\
\hline & 3 & 58 & 24 & 41 & 2,591 & 40,22 & 0,6715 & 0,6694 \\
\hline & 4 & 67 & 24 & 36 & 2,589 & 40,20 & 0,6718 & 0,6693 \\
\hline & 5 & 76 & 23 & 30 & 2,584 & 40,16 & 0,6725 & 0,6696 \\
\hline \multirow{4}{*}{$\mathrm{ST}+\mathrm{B}(\mathrm{ST})$} & 2 & 96 & 22 & 23 & 2,536 & 39,58 & 0,6785 & 0,6750 \\
\hline & 3 & 114 & 30 & 26 & 2,524 & 39,53 & 0,6800 & 0,6758 \\
\hline & 4 & 132 & 28 & 21 & 2,520 & 39,48 & 0,6805 & 0,6757 \\
\hline & 5 & 150 & 27 & 18 & 2,515 & 39,43 & 0,6811 & 0,6756 \\
\hline
\end{tabular}

${ }^{\mathrm{N} I}=$ Número de intervalos do polinômio B-spline quadrático; NP = Número de parâmetros; NPS = Número de parâmetros significativos $(\mathrm{p}<0,1)$; PPS = Porcentagem de parâmetros significativos $(\%)$; SQR = Soma de quadrados dos resíduos $\left(\times 10^{-7} \mathrm{~g}^{2}\right)$; DMA = Desvio médio absoluto $(\mathrm{g}) ; R^{2}=$ Coeficiente de determinação; $R_{a}^{2}=$ Coeficiente de determinação ajustado. ${ }^{2} \mathrm{~S}=$ efeito classificatório de sexo; $\mathrm{T}=$ efeito classificatório de tanque de criação; $\mathrm{ST}=$ efeitos classificatórios de sexo, tanque e interação sexo x tanque; $\mathrm{B}=\mathrm{B}$-spline sem aninhamento; $\mathrm{B}(\mathrm{S})=\mathrm{B}$-spline com aninhamento em sexo; $\mathrm{B}(\mathrm{T})=\mathrm{B}$-spline com aninhamento em tanque; $\mathrm{B}(\mathrm{ST})=\mathrm{B}$-spline com aninhamento na interação sexo $\mathrm{x}$ tanque.

Para rendimento de carcaça, à medida que os modelos foram se tornando mais parametrizados, os valores de SQR e DMA variaram pouco (Tab. 2). As diferenças entre os maiores e menores valores obtidos para esses critérios foram, respectivamente, de 0,261 e 0,224 . Os valores de NP, NPS, $R^{2}$ e $R_{a}^{2}$ foram os que apresentaram maior variação, de 16 a 150, 13 a 39, 0,1805 a 0,3525 e de 0,1745 a 0,3015 , respectivamente.
Considerando cada modelo estatístico testado para descrição das trajetórias médias do peso vivo e do rendimento de carcaça quanto ao arranjo dos efeitos de sexo, tanque de criação e aninhamento do polinômio B-spline a esses efeitos, o melhor modelo foi o $\mathrm{ST}+\mathrm{B}(\mathrm{ST})$, que incluiu os fatores sexo, tanque de criação e sua interação somados ao polinômio B-spline quadrático aninhado a essa interação (Tab. 1 e 2). 
Esse modelo foi considerado como de melhor ajustamento em função dos menores valores obtidos para SQR e DMA, e maiores valores de $R^{2}$ e $R_{a}^{2}$.

A inclusão da interação sexo $\mathrm{x}$ tanque e do aninhamento dos polinômios B-spline quadráticos a essa interação promoveu melhora no ajuste dos modelos estatísticos, indicando que essa interação foi importante, devendo ser considerada. A existência da interação sexo $\mathrm{x}$ tanque demonstrou que animais de sexos e tanques distintos cresceram de maneira diferenciada com o decorrer do tempo, possivelmente em função de fatores genéticos e ambientais. Essa interação indicou a existência de trajetórias médias de peso vivo e de rendimento de carcaça diferentes para cada sexo dentro de cada tanque. A inclusão dessa interação nos modelos é necessária para que os valores genéticos dos indivíduos, em avaliações genéticas futuras, sejam estimados de maneira correta. Considerar o aninhamento do efeito da idade em cada nível da interação sexo x tanque é importante para que indivíduos de determinado sexo, cultivados em determinado tanque, não sejam privilegiados durante a avaliação genética. Caso essa interação não seja incluída, animais de determinado sexo que foram cultivados em determinado tanque poderão ter os valores genéticos sub ou superestimados em alguma idade de avaliação, o que pode contribuir para alterar a classificação dos candidatos à seleção.

Tabela 2. Estatísticas de ajuste ${ }^{1}$ de polinômios B-splines quadráticos para a trajetória média do rendimento de carcaça de tilápias do Nilo (Oreochromis niloticus)

\begin{tabular}{|c|c|c|c|c|c|c|c|c|}
\hline Modelo $^{2}$ & NI & NP & NPS & PPS & SQR & DMA & $R^{2}$ & $R_{a}^{2}$ \\
\hline \multirow{4}{*}{$\mathrm{S}+\mathrm{T}+\mathrm{B}$} & 2 & 16 & 13 & 81 & 1,244 & 1,846 & 0,1805 & 0,1745 \\
\hline & 3 & 17 & 14 & 82 & 1,232 & 1,834 & 0,1889 & 0,1825 \\
\hline & 4 & 18 & 14 & 78 & 1,226 & 1,832 & 0,1924 & 0,1857 \\
\hline & 5 & 19 & 17 & 89 & 1,224 & 1,833 & 0,1939 & 0,1868 \\
\hline \multirow{4}{*}{$\mathrm{ST}+\mathrm{B}$} & 2 & 27 & 18 & 67 & 1,224 & 1,824 & 0,1943 & 0,1839 \\
\hline & 3 & 28 & 18 & 64 & 1,212 & 1,812 & 0,2018 & 0,1911 \\
\hline & 4 & 29 & 19 & 66 & 1,207 & 1,809 & 0,2052 & 0,1941 \\
\hline & 5 & 30 & 22 & 73 & 1,205 & 1,811 & 0,2066 & 0,1952 \\
\hline \multirow{4}{*}{$\mathrm{S}+\mathrm{T}+\mathrm{B}(\mathrm{S})$} & 2 & 19 & 15 & 79 & 1,196 & 1,803 & 0,2123 & 0,2053 \\
\hline & 3 & 21 & 17 & 81 & 1,186 & 1,794 & 0,2192 & 0,2114 \\
\hline & 4 & 23 & 19 & 83 & 1,179 & 1,789 & 0,2234 & 0,2150 \\
\hline & 5 & 25 & 20 & 80 & 1,175 & 1,788 & 0,2259 & 0,2167 \\
\hline \multirow{4}{*}{$\mathrm{S}+\mathrm{T}+\mathrm{B}(\mathrm{T})$} & 2 & 49 & 21 & 43 & 1,126 & 1,755 & 0,2584 & 0,2405 \\
\hline & 3 & 58 & 23 & 40 & 1,100 & 1,735 & 0,2753 & 0,2545 \\
\hline & 4 & 67 & 18 & 27 & 1,103 & 1,739 & 0,2739 & 0,2496 \\
\hline & 5 & 76 & 29 & 38 & 1,084 & 1,718 & 0,2864 & 0,2592 \\
\hline \multirow{4}{*}{$\mathrm{ST}+\mathrm{B}(\mathrm{ST})$} & 2 & 96 & 41 & 43 & 1,040 & 1,673 & 0,3152 & 0,2818 \\
\hline & 3 & 114 & 36 & 32 & 1,016 & 1,655 & 0,3307 & 0,2915 \\
\hline & 4 & 132 & 34 & 26 & 1,005 & 1,644 & 0,3382 & 0,2928 \\
\hline & 5 & 150 & 39 & 26 & 0,983 & 1,622 & 0,3525 & 0,3015 \\
\hline
\end{tabular}

${ }^{\mathrm{NI}}$ = Número de intervalos do polinômio B-spline quadrático; NP = Número de parâmetros; NPS = Número de parâmetros significativos $(\mathrm{p}<0,1)$; PPS = Porcentagem de parâmetros significativos $(\%)$; $\mathrm{SQR}=$ Soma de quadrados dos resíduos $\left(\mathrm{x} 10^{2} \%^{2}\right)$; DMA = Desvio médio absoluto $(\%) ; R^{2}=$ Coeficiente de determinação; $R_{a}^{2}=$ Coeficiente de determinação ajustado. ${ }^{2} \mathrm{~S}=$ efeito classificatório de sexo; $\mathrm{T}=$ efeito classificatório de tanque de criação; $\mathrm{ST}=$ efeitos classificatórios de sexo, tanque e interação sexo x tanque; $\mathrm{B}=\mathrm{B}$-spline sem aninhamento; $\mathrm{B}(\mathrm{S})$ = B-spline com aninhamento em sexo; $\mathrm{B}(\mathrm{T})=\mathrm{B}$-spline com aninhamento em tanque; $\mathrm{B}(\mathrm{ST})=\mathrm{B}$-spline com aninhamento na interação sexo $\mathrm{x}$ tanque.

O estudo das curvas de crescimento animal tem sido realizado no sentido de melhorar a eficiência da produção animal. Entretanto, diferenças dessas curvas em peixes, especificamente em linhagens de tilápias, não têm sido estudadas. No ajuste de funções de 
crescimento, é importante destacar que a forma das curvas pode apresentar variações entre diferentes raças e, dentro de uma mesma raça, entre o sexo dos animais. Além disso, o surgimento de diferentes linhagens de uma espécie de interesse zootécnico requer avaliação em diferentes ambientes e sistemas de cultivo (Santos et al., 2007a).

Apesar de o modelo ST+B(ST) ter sido escolhido como o mais adequado para estudar o peso vivo e o rendimento de carcaça dos indivíduos avaliados, ele foi o que apresentou o maior NP e a menor PPS (Tab. 1 e 2). Sabe-se que um parâmetro pode não ser significativo quando incluído isoladamente a um modelo, mas a sua inclusão, juntamente com outros parâmetros, pode melhorar os critérios de ajuste, reduzindo os valores de SQR e DMA e aumentando os valores de $R^{2}$ e $R_{a}^{2}$. Nos modelos mais parametrizados, foram incluídos parâmetros que não foram significativos, o que gerou baixo valor de PPS. Porém, nesses modelos, existem parâmetros significativos de alta importância que foram omitidos nos modelos mais simples que apresentaram alta PPS.

Considerando o melhor modelo estatístico escolhido $(\mathrm{ST}+\mathrm{B}(\mathrm{ST})$ ) e partindo para a escolha do número de intervalos dos polinômios B-spline quadráticos, nota-se que o modelo com cinco intervalos equidistantes (modelo com maior número de intervalos) foi considerado como o mais adequado para a análise de ambas as características, segundo os critérios SQR, DMA, $R^{2}$ e $R_{a}^{2}$ (Tab. 1 e 2 ).

As funções spline podem ser modeladas de várias formas (diferentes bases, posição dos nós e números de intervalos) e, dependendo da escolha, elas podem proporcionar redução da multicolinearidade, ser de fácil estimação e possuir interpretação biológica simples (Rey, 2008). Nas funções B-spline, pode-se observar que cada polinômio (função base) diferente de zero, presente entre dois nós, proporciona melhor qualidade de ajuste local, uma vez que o ajuste em determinado intervalo de idades é influenciado apenas por dados próximos a esse polinômio, e não por todo o conjunto de dados. Sendo assim, quanto maior for o número de intervalos, maior será o número de funções base e, consequentemente, melhor será o ajuste dos polinômios. Quanto maior o número de intervalos, maior o número de parâmetros requerido. Se maior quantidade de parâmetros resultar em maior aproximação dos valores estimados dos valores paramétricos, isso será consideravelmente vantajoso.

Os valores de $R^{2}$ e $R_{a}^{2}$ para peso vivo variaram respectivamente de 0,6574 a 0,6811 e de 0,6568 a 0,6756 em todos os modelos estatísticos estudados (Tab. 1). Tais valores foram considerados altos, indicando que as variáveis independentes incluídas explicaram a variação total observada para a característica de maneira satisfatória. Para rendimento de carcaça, em todos os modelos testados, os valores de $R^{2}$ e $R_{a}^{2}$ foram considerados baixos, indicando que outros fatores importantes, mas desconhecidos, não foram incluídos nos modelos utilizados e podem explicar parte da variabilidade para o rendimento de carcaça (Tab. 2).

Para peso vivo, os modelos $\mathrm{S}+\mathrm{T}+\mathrm{B}$ e $\mathrm{ST}+\mathrm{B}$ com cinco intervalos apresentaram os maiores valores de $R_{a}^{2}$ (Tab. 2). Para o modelo $\mathrm{S}+\mathrm{T}+\mathrm{B}(\mathrm{S})$, os modelos com quatro e cinco intervalos apresentaram os mesmos valores, ambos superiores aos valores dos modelos com dois e três intervalos (Tab. 2). Para rendimento de carcaça, os cinco modelos com cinco intervalos apresentaram os maiores valores de $R_{a}^{2}$ (Tab. 2).

Para peso vivo e rendimento de carcaça, o modelo $\mathrm{S}+\mathrm{T}+\mathrm{B}(\mathrm{T})$ com cinco intervalos apresentou maior valor de $R_{a}^{2}$, entretanto o modelo com três intervalos apresentou valor superior ao do modelo com quatro intervalos (Tab. 1 e 2).

Para peso vivo, o modelo $\mathrm{ST}+\mathrm{B}(\mathrm{ST})$ com três intervalos apresentou valor para $R_{a}^{2}$ superior aos demais pela inclusão de variáveis pouco explicativas (Tab. 1). Os modelos com quatro e cinco intervalos foram penalizados ao calcular-se o $R_{a}^{2}$, o que resultou em valores inferiores ao obtido no modelo com três intervalos. Entretanto, os critérios SQR, DMA e $R^{2}$ foram melhores para os modelos com maior número de intervalos, demonstrando que o aumento do 
número de intervalos faz com que haja melhor ajuste local em intervalos mais curtos de idade. Isso torna o modelo $\mathrm{ST}+\mathrm{B}(\mathrm{ST})$ com cinco intervalos o mais adequado.

Da mesma maneira que foram modestas as diferenças nos critérios de ajuste calculadas para os modelos com diferentes números de intervalos, os valores estimados de peso vivo e rendimento de carcaça ao longo do intervalo de idades também foram próximos.

Tanque 9

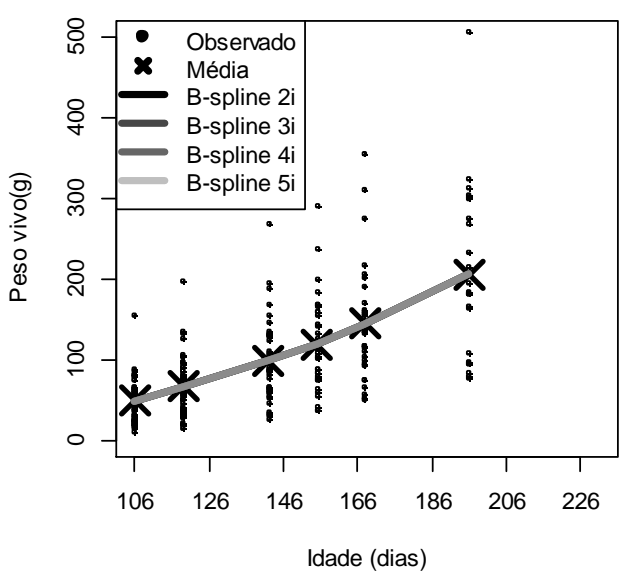

Tanque 9

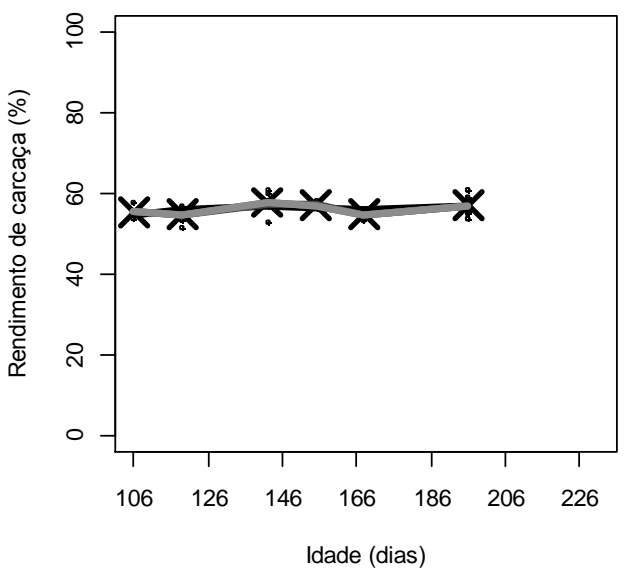

A análise das trajetórias médias do peso vivo estimadas com polinômios B-splines quadráticos de dois a cinco intervalos para machos nos tanques nove e dois, que apresentaram, respectivamente, menor e maior número de observações, demonstrou que não houve grandes diferenças entre os polinômios no decorrer das idades (Fig. 2). $\mathrm{O}$ fato de esses tipos de polinômios priorizarem o ajuste local em relação ao global os torna mais robustos em relação ao reduzido número de dados para certas faixas do conjunto de dados (Menezes et al., 2011).

Tanque 2

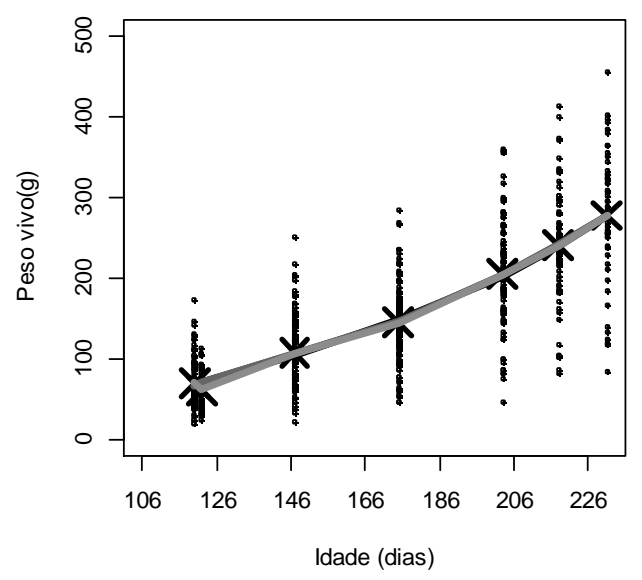

Tanque 2

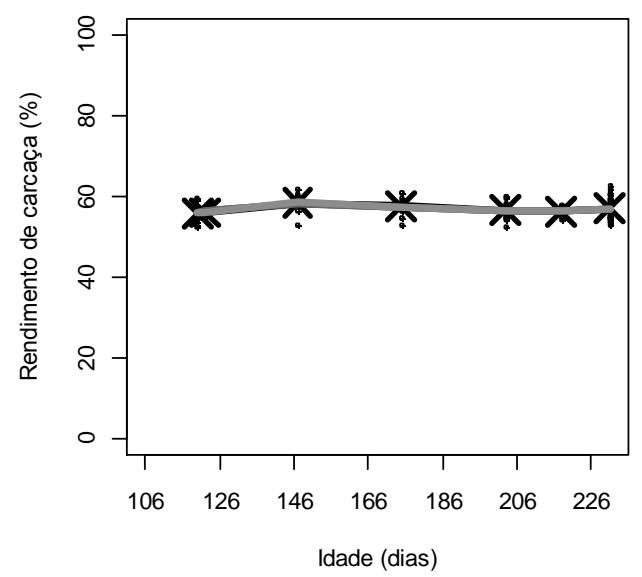

Figura 2. Trajetórias médias do peso vivo e do rendimento de carcaça nos tanques nove e dois com menor e maior número de observações, respectivamente, de machos de tilápias do Nilo (Oreochromis niloticus) estimadas por polinômios B-splines quadráticos com dois a cinco intervalos.

Para animais machos do tanque com maior número de observações, maiores diferenças entre as trajetórias do peso vivo puderam ser visualizadas entre a primeira e a segunda avaliação. Essas maiores oscilações dos polinômios nesses pontos podem ser explicadas pelo número de observações ter reduzido de 84 na primeira idade de avaliação para 44 na 
segunda. O polinômio B-spline quadrático com cinco intervalos foi o polinômio que mais se aproximou da média de peso vivo na segunda idade de avaliação dos animais machos do tanque 2 .

Não houve diferenças consideráveis entre os polinômios na análise das trajetórias médias do rendimento de carcaça (Fig. 2). Maiores diferenças foram visualizadas entre o polinômio B-spline quadrático com dois intervalos e os demais polinômios com três, quatro e cinco intervalos para machos do tanque com menor número de observações. Tais diferenças podem ser justificadas pelo fato de que, nas seis idades de avaliação desse tanque, foram obtidas poucas observações de rendimento de carcaça (quatro, seis, cinco, seis, seis e 24).

\section{CONCLUSÕES}

A inclusão da interação dos fatores sexo e tanque de criação e o aninhamento do efeito da idade, por meio de polinômios B-spline quadráticos, contribuíram para a melhoria dos ajustes dos modelos estatísticos para análise do peso vivo e do rendimento de carcaça de tilápias do Nilo (Oreochromis niloticus). $\mathrm{O}$ aumento do número de intervalos dos polinômios B-spline quadráticos melhorou o ajuste dos modelos estatísticos para análise do peso vivo e do rendimento de carcaça.

\section{REFERÊNCIAS}

BALDI, F.; ALENCAR M.M.; ALBUQUERQUE, L.G. Random regression analyses using B-splines functions to model growth from birth to adult age in Canchim cattle. J. Anim. Breed. Genet., v.127, p.433441, 2010.

BOLIGON, A.A.; MERCADANTE, M.E.Z.; LÔBO, R.B. et al. Random regression analyses using B-spline functions to model growth of Nellore cattle. Animal, v.6, p.212-220, 2012.

MENEZES, G.R.O.; TORRES, R.A.; TORRES JÚNIOR, R.A.A. et al. Modelo de regressão aleatória usando polinômios segmentados do tipo B no estudo da curva de crescimento em bovinos da raça Tabapuã. In: REUNIÃO ANUAL DA SOCIEDADE BRASILEIRA DE ZOOTECNIA, 48., 2011, Belém. Anais... Belém: SBZ, 2011. (CD-ROM).
MEYER, K. Random regression analyses using Bsplines to model growth of Australian Angus cattle. Genet. Sel. Evol., v.37, p.473-500, 2005.

REY, F.S.B. Estimação de parâmetros genéticos para características de crescimento em bovinos da raça Canchim com modelos de dimensão finita e infinita. 2008. 97f. Tese (Doutorado em Genética e Melhoramento Animal) - Universidade Estadual Paulista, Jaboticabal.

RUTTEN, M.J.M.; KOMEN, H.; BOVENHUIS, H. Longitudinal genetic analysis of Nile tilapia (Oreochromis niloticus L.) body weight using a random regression model. Aquaculture, v.246, p.101113, 2005.

SANTOS, D.C.C.; TORAL, F.L.B.; TURRA, E.M. et al. Fatores de variação para características de crescimento em Tilápias do Nilo (O. niloticus). In: CONGRESSO BRASILEIRO DE ZOOTECNIA, 22., 2012, Cuiabá. Anais... Cuiabá: ZOOTEC, 2012. (CDROM).

SANTOS, V.B.; FREITAS, R.T.F.; SILVA, F.F. et al. Avaliação de curvas de crescimento morfométrico de linhagens de tilápia do Nilo (Oreochromis niloticus). Ciênc. Agrotec., v.31, p.1486-1492, 2007a.

SANTOS, V.B.; FREITAS, R.T.F.; LOGATO, P.V.R. et al. Rendimento do processamento de linhagens de tilápias (Oreochromis niloticus) em função do peso corporal. Ciênc. Agrotec., v.31, p.554-562, $2007 \mathrm{~b}$.

SILVA, F.V.; SARMENTO, N.L.A.F.; VIEIRA, J.S. et al. Características morfométricas, rendimentos de carcaça, filé, vísceras e resíduos em tilápias-do-nilo em diferentes faixas de peso. Rev. Bras. Zootec., v.38, p.1407-1412, 2009.

TORAL, F.L.B.; PEREIRA, J.C.C.; BERGMANN, J.A.G. et al. Parâmetros genéticos do peso desde o nascimento até 730 dias de idade na raça Indubrasil. Pesq. Agropec. Bras., v.49, p.595-605, 2014.

TURRA, E.M.; OLIVEIRA, D.A.A.; VALENTE, B.D. et al. Longitudinal genetic analyses of fillet traits in Nile tilapia Oreochromis niloticus. Aquaculture, v.356-357, p.381-390, 2012.

VALENTE, B.D.; SILVA, M.A.; SILVA, L.O.C. et al. Estruturas de covariância de peso em função da idade de animais nelore das regiões sudeste e centro-oeste. Arq. Bras. Med. Vet. Zootec., v.60, p.389-400, 2008. 